\title{
A Comparison of the Incidence of Early Postoperative Infections between Patients Using Synthetic Mesh and Those Undergoing Traditional Pelvic Reconstructive Surgical Procedures
}

\author{
Mašata J. ${ }^{1}$, Martan A. ${ }^{1}$, Poislová M. ${ }^{2}$, Kobilková J. ${ }^{1}$, Mašatová D. ${ }^{2}$, \\ Jedličková A. ${ }^{2}$, Švabík K. ${ }^{1}$, Hubka P. ${ }^{1}$, Zvára K. ${ }^{3}$ \\ 1Department of Obstetrics and Gynaecology, First Faculty of Medicine, Charles \\ University in Prague and General University Hospital in Prague, Prague, Czech \\ Republic;
}

${ }^{2}$ Clinical Microbiology and Antibiotic Centre, Institute of Medical Biochemistry and Laboratory Diagnostics, First Faculty of Medicine, Charles University in Prague and General University Hospital in Prague, Prague, Czech Republic;

${ }^{3}$ EuroMISE Center, Institute of Computer Science, Academy of Sciences of the Czech Republic, Prague, Czech Republic

Received July 2, 2012; Accepted April 8, 2013.

Key words: Febrile morbidity - Pelvic organ prolapse - Postoperative infections Sepsis - Synthetic polypropylene mesh

\begin{abstract}
New mesh-related complications such as erosion, etc., can result from abnormal postoperative healing due to surgical site infection. The aim of our study was to compare systemic inflammatory responses and the incidence of early infectious complications after reconstructive surgery using synthetic mesh and after traditional vaginal wall repair. In this prospective observational study 99 women with symptomatic pelvic organ prolapse were included; 55 women underwent traditional repair and 44 repair using mesh. After the procedure infectious complications were monitored. The patients who underwent reconstructive
\end{abstract}

This study was supported by the Grant Agency of the Ministry of Health of the Czech Republic, grant NR/9441-3, GIGH-0751-00-3-223, and Charles University in Prague, project UNCE 204024.

Mailing Address: Assoc. Prof. Jaromír Mašata, MD., PhD., Department of Obstetrics and Gynaecology, First Faculty of Medicine, Charles University in Prague and General University Hospital in Prague, Apolinářská 18, 12000 Prague 2, Czech Republic; Mobile Phone: +420 603444 662; Fax: +420 224967 474; e-mail:masata@volny.cz 
surgery using mesh material were more likely to have febrile morbidity in the postoperative period than the patients who had been treated with traditional repair $(p=0.031)$; there was a higher incidence of combination febrile morbidity with elevated C-reactive protein (CRP) $>50 \mathrm{mg} / \mathrm{l} ; \mathrm{p}=0.046$, and a higher incidence of CRP increase over $30 \mathrm{mg} / \mathrm{l} ; \mathrm{p}=0.005$. Reconstructive procedures using synthetic mesh are accompanied by a higher incidence of early post-operative infectious complications.

\section{Introduction}

Pelvic organ prolapse is a common problem affecting up to $50 \%$ of parous women (Subak et al., 2001). Not all of them are symptomatic (Olsen et al., 1997; Baessler and Maher, 2006), and the risk of requiring surgery for prolapse is $11-12 \%$ in women reaching 79 years of age (Olsen et al., 1997). For a long time traditional pelvic reconstruction surgical procedures were the only ones available. The longterm efficiency of those procedures varied, and re-operation rates of nearly $30 \%$ have been reported by Olsen et al. (1997), with a risk of recurrence of up to $45 \%$ according to Valaitis and Stanton (1994) and Kohli et al. (1998). This may be one of the reasons why synthetic meshes were introduced to pelvic reconstructive surgery. However, these new surgical procedure have brought with them new mesh-related complications such as erosion, pain syndrome, etc. as described by Bako and Dhar (2009). Some of these complications - erosion, shrinkage and abnormal pain - can result from abnormal postoperative healing due to surgical site infection.

The vagina contains more microorganisms than any other site in the body except the bowel (Lamont and Haynes, 2008). Surgical procedures resulting in the opening of the vagina will result in contamination of normally sterile sites by bacteria that are normally resident in the vagina. Infection and inflammation depends on a mixture of surgical and host-related factors. When mesh material is used this decreases the size of the bacterial inoculum, which can induce postoperative infection.

The aim of our study was to compare immediate inflammatory reaction and the incidence of infectious complications after reconstructive surgery using synthetic macro-porous polypropylene mesh and after traditional vaginal wall repair.

\section{Material and Methods}

This prospective observational study included 99 women with symptomatic pelvic organ prolapse stage II or higher, according to Pelvic Organ Prolapse Quantitative (POP-Q) (Bump et al., 1996). All of them underwent surgical procedures between January 2007 and December 2008. The women were divided into two subgroups according to the type of procedure: 55 women underwent traditional vaginal wall repair (TR group) and 44 repair using mesh material (M group). Traditional 
repair was taken to include anterior colporrhaphy with fascial plication, posterior repair with levator plication and - for patients with apical defect - AmreichRichter procedure was added. Commercially available kits were used for mesh reconstruction (GYNECARE, PROLIFT ${ }^{\circledR}$ Pelvic Floor Repair System, Ethicon, Johnson and Johnson Company, NJ, USA). During the procedure we followed all recommended steps, including changing surgical gloves before manipulation with mesh and irrigation of the surgical field before mesh insertion with iodopovidonum (Betadine, EGIS Pharmaceuticals Ltd., Budapest, Hungary) or with octylamonium plus 2-fenoxyethan-1-ol (Octenisept, Schülke and Mayr GmbH, Germany) in patients with an allergy to iodine.

Concomitant hysterectomy was not an exclusion criterion. After the surgery each patient in both groups received a vaginal pack for 24 hours and a Foley catheter for 48 hours. The study was approved by the local ethics committee, and each woman signed an informed consent before enrolment. Operations were indicated consecutively, without randomization (all surgical procedures were performed by MJ, MA and SK).

Before surgery all patients received estriol (Ovestin, N.V. ORGANON, OSS, The Netherlands) intravaginally for six weeks at a minimum.

One day before surgery a detailed history was taken, focusing especially on factors increasing postoperative infections (host-related factors such as poor nutrition, obesity, diabetes, impaired immunocompetence, and others). Pelvic examination was performed, and the character of the vaginal discharge was assessed (i.e. colour, smell, with or without addition of $10 \% \mathrm{KOH}$ ).

Measurement of vaginal $\mathrm{pH}$ was made with colour $\mathrm{pH}$ strip paper $\mathrm{pH}$ range 4.0-7.0 (Merck, Germany) using vaginal secretions obtained from the lateral pelvic wall; a sample of vaginal fluid was obtained from the upper lateral vaginal vault and spread onto two glass slides (for Gram and Giemsa stain) and after fixation sent to the laboratory for microscopic examination to ascertain the presence of asymptomatic infection, especially bacterial vaginosis, aerobic vaginitis, atrophic vaginitis and Trichomonas vaginalis infection. The presence of bacterial vaginosis (BV) was evaluated based on Nugent's criteria (Nuget et al., 1991), while the presence of aerobic vaginitis was assessed using Donders' criteria (Donders et al., 2002).

To exclude differences in preoperative vaginal hormonal status another smear from the upper lateral vaginal vault was obtained, and after fixation this was sent to the cytological laboratory for Papanicolaou stain. Overall estrogenic activity was assessed using maturation index, and the presence of parabasal and basal epithelial cells was monitored as described by Kobilkova (1967) and Bibbo (1997).

Haemoglobin level and leukocyte count was also assessed one day before surgery.

Preoperatively all patients received prophylactic antibiotic Ampicilin+Sulbactam 1.5 g i.v. (Unasyn, Pfizer, CR) or Clindamycine 900 mg (Dalacin, Pfizer, CR) for patients allergic to penicillines, and a second dose 6 hours after the surgery; for 
Clindamycine the second dose was reduced to $600 \mathrm{mg}$. Careful peri-operative antisepsis of the perineum, vulva and vagina was carried out, including covering the anus. If there was any suspicion of infection further use of antibiotics was not restricted, and indication was left to the attending physician. Incidence of further antibiotic use was also compared in both groups.

After the procedure systemic inflammatory response parameters were monitored (pulse, temperature), and on the third day after the procedure a blood sample was taken for haemoglobin, leukocyte count and C-reactive protein (CRP) level, along with a urine sample and vaginal smear for cultivation. Blood loss was estimated on haemoglobin drop comparing pre-operative and postoperative haemoglobin levels.

Infections with a systemic inflammatory response were monitored: e.g. febrile morbidity sepsis and others (we used previously published definitions of various systemic inflammatory responses by Tamussino, 2002). All patients underwent vaginal examination before discharge or when a temperature higher than $38{ }^{\circ} \mathrm{C}$ was established after the third postoperative day. The presence of a foul-smelling discharge, erythema, purulent discharge or pain and tenderness at the surgical margins was classified as vaginal wound infection. In the case of an infection, a vaginal smear was taken for microbiological examination to assess the presence of bacteria and the bacterial susceptibility to antibiotics. If empirical antibiotic treatment failed, further treatment was modified according to the results of microbiological testing.

Prophylaxis against thromboembolism was given to all women, Enoxaparinum (Sanofi-Aventis, Prague, CR).

Before collecting the data we assumed $10 \%$ incidence of complication after traditional repair (TR), and we wanted to detect at least $25 \%$ difference in the increase of post-operative infectious complications (i.e. to detect at least $35 \%$ incidence of complications after mesh procedures $(M))$ with alpha $5 \%$ and power $80 \%$. Under these assumptions statistical calculations indicate that the required sample size in each group is 43 patients.

All statistical tests were performed at $5 \%$ level of significance. We used the nonparametric Mann-Whitney test and, where appropriate, chi-square test with Yates correction or Fisher exact test. Odds ratios and relative risk with corresponding $95 \%$ intervals of confidence were also calculated.

\section{Results}

The two groups do not differ in basic demographic characteristics (Table 1), in mean parity (TR group 2.05 ( $\mathrm{SD}=0.62)$ and $M$ group $2.07(\mathrm{SD}=0.82)$ ), in number of previous hysterectomies (59.1\% compared with $41.8 \%$ ) or in cases of reconstructive surgery ( $25 \%$ vs. $20 \%$ ) in their history (Table 1$)$.

Neither do the groups differ in any other preoperative characteristics; e.g. presence of internal diseases, hypertension, diabetes type II (DM II), vaginal 


\section{Table 1 - Pre-operative characteristics}

Table 1a

\begin{tabular}{lccc}
\hline & $\begin{array}{c}\text { TR } \\
(\text { mean/SD })\end{array}$ & $\begin{array}{c}\text { M } \\
(\text { mean/SD })\end{array}$ & $\mathrm{P}^{*}$ \\
\hline Age (years) & $61.46 / 11.95$ & $63.17 / 8.98$ & 0.39 \\
BMI & $27.11 / 4.30$ & $26.93 / 4.44$ & 0.91 \\
Hb level (g/l) & $135.89 / 10.40$ & $138.45 / 11.28$ & 0.20 \\
Leu 10^9/I & $6.70 / 1.76$ & $7.25 / 2.61$ & 0.21 \\
$\mathrm{PH}$ & $5.31 / 0.83$ & $5.06 / 0.74$ & 0.24 \\
$\mathrm{MI}$ & $53.75 / 27.57$ & $55.84 / 26.98$ & 0.65 \\
$\mathrm{BC}$ & $13.95 / 27.85$ & $9.07 / 24.84$ & 0.15 \\
\hline
\end{tabular}

*Mann-Whitney test; TR - traditional vaginal wall repair group; $\mathrm{M}$ - vaginal wall repair using mesh material group; $\mathrm{BMI}$ - body mass index; $\mathrm{Hb}$ - hemoglobin; Leu - leucocyte count; $\mathrm{MI}$ - maturation index; $\mathrm{BC}$ - presence of parabasal and basal cells on hormonal cytology

\section{Table 1b}

\begin{tabular}{lccc}
\hline & $\begin{array}{c}\text { TR } \\
(\mathrm{n} / \%)\end{array}$ & $\begin{array}{c}\mathrm{M} \\
(\mathrm{n} / \%)\end{array}$ & $\mathrm{P}^{*}$ \\
\hline Hysterectomy & $23 / 41.8$ & $26 / 59.1$ & 0.08 \\
Pelvic reconstruction & $11 / 20.0$ & $11 / 25.0$ & 0.72 \\
Any internal disease & $53 / 96.4$ & $39 / 88.6$ & 0.27 \\
Hypertension & $30 / 54.5$ & $16 / 36.4$ & 0.11 \\
DM II & $7 / 12.7$ & $7 / 15.9$ & 0.87 \\
$\mathrm{PH}>4.5$ & $49 / 90.7$ & $35 / 79.5$ & 0.19 \\
$\mathrm{BC}$ & $16 / 29.1$ & $7 / 15.9$ & 0.19 \\
Asymptomatic vag. inf. & $3 / 5.5$ & $4 / 9.1$ & 0.75 \\
Concomitant hysterectomy & $19 / 34.5$ & $11 / 25$ & 0.42 \\
\hline
\end{tabular}

$*^{2} \chi^{2}$ test (with Yates correction); TR - traditional vaginal wall repair group; $M$ - vaginal wall repair using mesh material group; DM II - diabetes type II; BC - presence of parabasal and basal cells on hormonal cytology

Table 2 - Post-operative characteristics

\begin{tabular}{|c|c|c|c|}
\hline & $\begin{array}{c}\text { TR } \\
\text { (mean/SD) }\end{array}$ & $\begin{array}{c}M \\
\text { (mean/SD) }\end{array}$ & $\mathrm{p}^{*}$ \\
\hline Hb level $(g / l)$ & $121.0 / 11.38$ & $113.55 / 9.96$ & 0.001 \\
\hline Leu $10^{\wedge} 9 / /$ & $7.84 / 2.54$ & $7.58 / 2.07$ & 0.89 \\
\hline $\mathrm{Hb}$ drop $(g / l)$ & $-14.89 / 11.06$ & $-24.91 / 12.19$ & 0.0001 \\
\hline CRP & $54.62 / 39.1$ & $60.60 / 28.84$ & 0.08 \\
\hline Operation time (minutes) & $74.27 / 27.47$ & $83.93 / 28.06$ & 0.07 \\
\hline
\end{tabular}

${ }^{*} \chi^{2}$ test (with Yates correction); TR - traditional vaginal wall repair group $(n=55)$; $M$ - vaginal wall repair using mesh material group $(n=44) ; \mathrm{Hb}$ - hemoglobin; Leu - leucocyte count; CRP - C-reactive protein 
Table 3 - Comparison of post-operative infectious complication

\begin{tabular}{lcccccr}
\hline & $\begin{array}{c}\text { TR } \\
(\mathrm{n} / \%)\end{array}$ & $\begin{array}{c}\mathrm{M} \\
(\mathrm{n} / \%)\end{array}$ & $\mathrm{P}^{*}$ & OR & RR & Cl 95\% \\
\hline CRP $>30$ & $33 / 62.3$ & $39 / 88.6$ & $\mathbf{0 . 0 0 5}$ & 4.65 & 1.42 & $1.13-1.80$ \\
ATB & $14 / 25.5$ & $20 / 45.5$ & 0.055 & 2.42 & 1.79 & $1.02-3.11$ \\
FM & $8 / 14.5$ & $15 / 34.1$ & $\mathbf{0 . 0 3 1}$ & 3.00 & 2.34 & $1.09-5.02$ \\
FM+CRP > 50 & $7 / 12.7$ & $13 / 29.5$ & $\mathbf{0 . 0 4 6}$ & 2.84 & 2.32 & $1.01-5.32$ \\
Urine & $6 / 10.9$ & $6 / 13.6$ & 0.762 & 1.29 & 1.25 & $0.43-3.61$ \\
\hline
\end{tabular}

*Fisher exact test; TR - traditional vaginal wall repair group $(n=55) ; M$ - vaginal wall repair using mesh material group ( $n=44$ ); CRP - C-reactive protein; ATB - further antibiotic use; FM - febrile morbidity; FM+CRP - febrile morbidity plus elevated CRP; Urine - positive urine culture (presence of colony - forming units $\geq 10^{5}$ ); OR - odds ratio; $\mathrm{RR}$ - relative risk; $\mathrm{Cl}$ - confidence interval

hormonal status, vaginal $\mathrm{pH}$, presence of asymptomatic vaginal infection and blood count (Table 1).

In the M group 9 patients underwent total Prolift, while the rest had anterior or posterior Prolift. In the traditional repair group 35 patients underwent anterior and posterior vaginal wall repair, while the rest of the patients had one of these procedures; for ten patients the Amreich-Richter procedure was added. There is also no difference between the groups in terms of presence of concomitant hysterectomy (TR 19/34.5\%, M 11/25\%) and mean operation time (Table 2). However, in the mesh group higher blood loss was noted based on post-operative haemoglobin drop (Table 2). None of the patients required blood transfusion.

After reconstructive surgery using mesh material patients have a higher risk in the postoperative period of having febrile morbidity than patients who underwent traditional repair, $\mathrm{p}=0.031$ (Table 3). In the mesh group there was also a higher incidence of combination febrile morbidity with elevated CRP $>50 \mathrm{mg} / \mathrm{l} ; \mathrm{p}=0.046$. CRP increase over $30 \mathrm{mg} / \mathrm{l}$ was more frequently present in the mesh group; $p=0.005$ (Table 3), but no differences in mean CRP levels were noted. In one case after the traditional procedure vaginal wound infection was noted; in contrast there were 4 cases after mesh repair. This incidence is too low to produce a statically significant difference. Incidence of further antibiotic use was also higher in the mesh group; the difference is significant clinically but not statistically (Table 3 ). There was no difference in the occurrence of postoperative urinary tract infection. All infections were mild or moderate; in none of the patients was abscess formation or severe sepsis observed, and no readmission was noted.

\section{Discussion}

In recent years there has been an increase in the use of synthetic meshes in pelvic floor reconstructive surgery. Simultaneously there is increasing evidence of long term mesh-related complications described by Bako and Dhar (2009). These complications could have significant impact on the quality of life of those patients 
according to Ridgeway et al. (2008). Complications could be caused by the surgical technique or the type of mesh. In our study we used macro-porous mesh Type I using Amid classification (Amid, 1997). This type of mesh is considered to have a lower risk of infection. Different biomaterials used in vaginal surgery have different bacterial infectiosity (Mathe et al., 2007). Studies on animal models show that the link between infections may play an important role in mesh related complication. Non-infected synthetic meshes were less likely to contract than infected meshes. Polypropylene mesh also induces mild but persistent foreign body reaction (Elmer et al., 2009b).

Regarding surgical technique; all procedures were performed by senior surgeons with more than 10 years surgical experience who had been using mesh for pelvic reconstruction procedures for more than 5 years. Patients undergoing pelvic reconstructive surgery are candidates for antibiotic prophylaxis. This is reasonable in that the vaginal epithelium is incised and can be considered a cleancontaminated procedure by surgical wound classification. No prospective studies have been performed in this setting. Prophylactic antibiotics are routinely used in studies evaluating the effectiveness of a sling, including those using a mesh placed transvaginally (Altman and Falconer, 2007; Elmer et al., 2009a), despite the lack of randomized clinical trials. There is also no evidence of comparison of early infectious complication between patients using a mesh and those after traditional pelvic reconstructive surgery.

It is hard to assess the real prevalence of postoperative infection. Nowadays the situation is complicated. It is ethically unacceptable to perform studies where prophylactic antibiotics are not used (Mittendorf et al., 1993). A further issue is whether prophylactic antibiotics for mesh procedure should be the same as for vaginal hysterectomy and traditional reconstructive surgery, or should be different. Another problem is abundant antibiotic use in the postoperative period. There is a fear of infectious complications and the desire not to prolong the patient's stay in hospital. In our study there is also relatively higher postoperative use of antibiotics, which is higher in the mesh group (the difference is clinically important but not statistically significant). The antibiotic use does not correlate with the prevalence of objective infectious markers, which are lower.

Because our study was not randomized, we looked at all differences in factors which can increase the rate of postoperative infectious complications. The two groups do not differ in age, BMI, parity, presence of internal diseases (DM II, hypertension), previous reconstructive surgery or hysterectomies in their history. We focused on the presence of asymptomatic vaginal infections which can increase the postoperative infectious complication rate (Soper et al., 1990; Larsson et al., 1991). The prevalence of asymptomatic vaginal infections in our group was low, and there was no correlation to postoperative infectious morbidity.

The risk of postoperative infection also depends on the virulence and size of the bacterial inoculum. Postmenopausal decrease in oestrogen levels leads to a 
marked change in vaginal flora. Lactobacillus spp. are absent, or present at only low levels, and the vaginal $\mathrm{pH}$ becomes elevated. There is concomitant increase in colonization by Gram-positive cocci and coliforms in the vagina. The use of hormone replacement therapy by post-menopausal women results in a return to a Lactobacillus-dominated vaginal flora (Pabich et al., 2003). Although all the patients in our group received estriol locally, a large proportion of them had elevated vaginal $\mathrm{pH}$. This fact did not negatively influence the traditional repair group; febrile morbidity in this group did not differ from that reported by Hirsh (1985) after vaginal hysterectomy using antibiotic prophylaxis. But after the mesh procedure the presence of those potentially pathogenic bacteria may represent sufficient infectious inoculums (despite the use of antibiotic prophylaxis and proper asepsis) to induce postoperative infection. It is possible that estriol application twice weekly before mesh surgery is not sufficient to improve the vaginal environment; possibly more intensive application is needed.

Persistent CRP elevation after surgery can be predictive of infectious postoperative complications. CRP exhibits an exponential rise in serum concentration following an acute insult, followed by an almost equally rapid descent to near-normal levels after termination of the stimulus. The normal serum level is less than $10 \mathrm{mg} / \mathrm{l}$. Following trauma CRP serum levels rise to greater than $100 \mathrm{mg} / \mathrm{l}$ within 24 hours (Mustard et al., 1987; Welsch et al., 2007). The rise is exponential with a doubling time of eight to nine hours. In uneventful postoperative course CRP serum levels start returning to normal levels on the third day after surgery. We cannot exclude the possibility that elevated CRP serum levels in some cases in the mesh group may only reflect more extensive dissection before mesh placement and local reaction to mesh, but there is no difference in mean CRP levels. On the other hand, CRP third day elevation over $50 \mathrm{mg} / \mathrm{l}$ accompanies febrile morbidity.

Fever is the most common symptom of postoperative infection. Other reasons for fever should be ruled out, such as tissue injury, drug reactions or atelectasis (Tamussino, 2002). Antibiotic therapy should not be employed for fever alone (Wittmann and Schein, 1996). In some cases the diagnosis is made by the attending physician, without apparent criteria. Antibiotic use in our study did not correlate with prevalence of objective infectious markers.

Prevalence of postoperative urinary tract infection in our group does not differ from other studies, where Ampicilin+Sulbactam was used for antibiotic prophylaxis (Falagas et al., 2008).

The available studies mainly deal with those mesh-related infections which occur later after surgery. The incidence of those infections ranged from 0 to $8 \%$ according to studies by Deffieux et al. (2007), de Tayrac et al. (2007) and Falagas et al. (2007). Various Gram-positive and Gram-negative aerobic and anaerobic bacteria have been identified in this complication. Peri-operative bacterial colonisation and abnormal postoperative healing due to infection may also be one of the most important factors in the development of other mesh-related complications. 
A Scandinavian study by Altman and Falconer (2007) described low prevalence of perioperative infectious complications, but in the same patient cohort $11 \%$ of mesh erosion was described by Elmer et al. (2009a). In our study mesh erosion occurred in 4 patients; 2 of them have moderate postoperative infection, one mild and the last one had no signs of postoperative infection.

Further prospective randomized studies are necessary to compare the occurrence of early postoperative infectious morbidity and long term complication such as erosion, abnormal pain, etc. More studies dealing with a comparison between the effect of different prophylactic antibiotic regimens or prolonged antibiotic treatment on the incidence of postoperative infection and their sequelae are also needed.

\section{Conclusion}

After reconstructive surgery using mesh material patients have a higher risk in the postoperative period of having febrile morbidity than patients who underwent traditional repair, $p=0.03$. In the mesh group there was also a higher incidence of combination febrile morbidity with elevated CRP > $50 \mathrm{mg} / \mathrm{l} ; \mathrm{p}=0.046$. CRP increase over $30 \mathrm{mg} / \mathrm{l}$ was more frequently present in the mesh group; $p=0.005$, but no differences in mean CRP levels were noted. In one case after the traditional procedure vaginal wound infection was noted; in contrast there were 4 cases after mesh repair. This incidence is too low to produce a statically significant difference. Incidence of further antibiotic use was also higher in the mesh group; the difference is significant clinically but not statistically. There was no difference in the occurrence of postoperative urinary tract infection. All infections were mild or moderate; in none of the patients was abscess formation or severe sepsis observed, and no readmission was noted.

\section{References}

Altman, D., Falconer, C. (2007) Perioperative morbidity using transvaginal mesh in pelvic organ prolapse repair. Obstet. Gynecol. 109, 303-308.

Amid, P. (1997) Classification of biomaterials and their related complication in abdominal wall hernia surgery. Hernia 1, 15-21.

Baessler, K., Maher, C. F. (2006) Mesh augmentation during pelvic-floor reconstructive surgery: risks and benefits. Curr. Opin. Obstet. Gynecol. 18, 560-566.

Bako, A., Dhar, R. (2009) Review of synthetic mesh-related complications in pelvic floor reconstructive surgery. Int. Urogynecol. J. Pelvic Floor Dysfunct. 20, 103-111.

Bibbo, M. (1997) Comprehensive Cytopathology. W.B. Saunders Co., Philadelphia.

Bump, R., Mattiasson, A., Bo, K., Brubaker, L., DeLancey, J., Klarskov, P., Shull, B., Smith, A. (1996) The standardization of terminology of female pelvic organ prolapse and pelvic floor dysfunction. Am. J. Obstet. Gynecol. 175, 10-17.

Deffieux, X., de Tayrac, R., Huel, C., Bottero, J., Gervaise, A., Bonnet, K., Frydman, R., Fernandez, H. (2007) Vaginal mesh erosion after transvaginal repair of cystocele using Gynemesh or Gynemesh-Soft in 138 women: a comparative study. Int. Urogynecol. J. Pelvic Floor Dysfunct. 18, 73-79. 
de Tayrac, R., Devoldere, G., Renaudie, J., Villard, P., Guilbaud, O., Eglin, G. (2007) Prolapse repair by vaginal route using a new protected low-weight polypropylene mesh: 1-year functional and anatomical outcome in a prospective multicentre study. Int. Urogynecol. J. Pelvic Floor Dysfunct. 18, 251-256.

Donders, G., Vereecken, A., Bosmans, E., Dekeersmaecker, A., Salembier, G., Spitz, B. (2002) Definition of a type of abnormal vaginal flora that is distinct from bacterial vaginosis: aerobic vaginitis. BJOG 109, 34-43.

Elmer, C., Altman, D., Engh, M. E., Axelsen, S., Vayrynen, T., Falconer, C. (2009a) Trocar-guided transvaginal mesh repair of pelvic organ prolapse. Obstet. Gynecol. 113, 117-126.

Elmer, C., Blomgren, B., Falconer, C., Zhang, A., Altman, D. (2009b) Histological inflammatory response to transvaginal polypropylene mesh for pelvic reconstructive surgery.J. Urol. 181, 1189-1195.

Falagas, M. E., Velakoulis, S., lavazzo, C., Athanasiou, S. (2007) Mesh-related infections after pelvic organ prolapse repair surgery. Eur. J. Obstet. Gynecol. Reprod. Biol. 134, 147-156.

Falagas, M. E., Athanasiou, S., lavazzo, C., Tokas, T., Antsaklis, A. (2008) Urinary tract infections after pelvic floor gynecological surgery: prevalence and effect of antimicrobial prophylaxis. A systematic review. Int. Urogynecol. J. Pelvic Floor Dysfunct. 19, 1165-1172.

Hirsch, H. A. (1985) Prophylactic antibiotics in obstetrics and gynecology. Am. J. Med. 78, 170-176.

Kobilkova, J. (1967) Cytologic study of the level of estrogen during the reproductive age. Acta Cytol. 11, 497-500.

Kohli, N., Walsh, P. M., Roat, T. W., Karram, M. M. (1998) Mesh erosion after abdominal sacrocolpopexy. Obstet. Gynecol. 92, 999-1004.

Lamont, R. F., Haynes, S. V. Z. (2008) Prevention of infection following gynecological surgery: the evidence. In: Complications in Gynecological Surgery. O’Donovan, P., Pp. 1-10, Springer, London.

Larsson, P. G., Platz-Christensen, J. J., Forsum, U., Pahlson, C. (1991) Clue cells in predicting infections after abdominal hysterectomy. Obstet. Gynecol. 77, 450-452.

Mathé, M. L., Lavigne, J. P., Oliva-Lauraire, M. C., Guiraud, I., Marès, P., de Tayrac, R. (2007) Comparison of different biomaterials for vaginal surgery using an in vivo model of meshes infection in rats. Gynecol. Obstet. Fertil. 35, 398-405. (in French)

Mittendorf, R., Aronson, M. P., Berry, R. E., Williams, M. A., Kupelnick, B., Klickstein, A., Herbst, A. L., Chalmers, T. C. (1993) Avoiding serious infections associated with abdominal hysterectomy: a metaanalysis of antibiotic prophylaxis. Am. J. Obstet. Gynecol. 169,1119-1124.

Mustard, R. A. Jr., Bohnen, J. M., Haseeb, S., Kasina, R. (1987) C-reactive protein levels predict postoperative septic complications. Arch. Surg. 122, 69-73.

Nugent, R. P., Krohn, M. A., Hillier, S. L. (1991) Reliability of diagnosing bacterial vaginosis is improved by a standardized method of gram stain interpretation. J. Clin. Microbiol. 29, 297-301.

Olsen, A. L., Smith, V. J., Bergstrom, J. O., Colling, J. C., Clark, A. L. (1997) Epidemiology of surgically managed pelvic organ prolapse and urinary incontinence. Obstet. Gynecol. 89, 501-506.

Pabich, W. L., Fihn, S. D., Stamm, W. E., Scholes, D., Boyko, E. J., Gupta, K. (2003) Prevalence and determinants of vaginal flora alterations in postmenopausal women. J. Infect. Dis. 188, 1054-1058.

Ridgeway, B., Chen, C. C., Paraiso, M. F. (2008) The use of synthetic mesh in pelvic reconstructive surgery. Clin. Obstet. Gynecol. 51, 136-152.

Soper, D. E., Bump, R. C., Hurt, W. G. (1990) Bacterial vaginosis and trichomoniasis vaginitis are risk factors for cuff cellulitis after abdominal hysterectomy. Am. J. Obstet. Gynecol. 163, 1016-1021, discussion 1021-1023.

Subak, L. L., Waetjen, L. E., van den Eeden, S., Thom, D. H., Vittinghoff, E., Brown, J. S. (2001) Cost of pelvic organ prolapse surgery in the United States. Obstet. Gynecol. 98, 646-651.

Tamussino, K. (2002) Postoperative infection. Clin. Obstet. Gynecol. 45, 562-573.

Valaitis, S. R., Stanton, S. L. (1994) Sacrocolpopexy: a retrospective study of a clinician's experience. Br. J. Obstet. Gynaecol. 101, 518-522.

Mašata J.; Martan A.; Poislová M.; Kobilková J.; Mašatová D.; Jedličková A.; Švabík K.; Hubka P.; Zvára K. 
Welsch, T., Muller, S. A., Ulrich, A., Kischlat, A., Hinz, U., Kienle, P., Buchler, M. W., Schmidt, J., Schmied, B. M. (2007) C-reactive protein as early predictor for infectious postoperative complications in rectal surgery. Int. J. Colorectal Dis. 22, 1499-1507.

Wittmann, D. H., Schein, M. (1996) Let us shorten antibiotic prophylaxis and therapy in surgery. Am. J. Surg. 172, 26S-32S. 